\title{
Hypothyreose und Verhaltensauffälligkeiten beim Hund - Sind sie über- oder unterdiagnostiziert?
}

\author{
Christiane Wergowski
}

Die Schilddrüsenunterfunktion gehört zu den häufigsten, gleichzeitig aber auch zu den umstrittensten endokrinen Erkrankungen des Hundes. Dieser Artikel soll helfen, die Ursachen dieses Phänomens zu verstehen. Er beschreibt die Funktion der Schilddrüse sowie die verschiedenen Formen der Hypothyreose und geht dann ausführlich auf die autoimmune Thyreoiditis ein. Die Möglichkeiten der Diagnostik und Therapie einer subklinischen Hypothyreose werden erläutert und die aus Erfahrung der Autorin am häufigsten betroffenen Rassen aufgelistet.

\section{Physiologie der Schilddrüse Funktion}

Die Schilddrüse produziert in ihren Follikeln aus der Aminosäure Tyrosin und Jodmolekülen die Hormone Thyroxin (T4) und Trijodthyronin (T3). Die Schilddrüsenfollikel dienen gleichzeitig auch als Speicher für die Hormone. Von dort werden sie ins Blut abgegeben und in freier Form oder an Transportglobuline gebunden zu den Zielorganen transportiert.

\section{Regulation}

Gesteuert wird die Schilddrüsenfunktion durch eine Reihe von Regulationsmechanismen. Der Hypothalamus produziert TRH (Thyreotropin Releasing Hormon), das auf die Hypophyse wirkt und dort die Ausschüttung von TSH (Thyreoidea-stimulierendes Hormon, Thyreotropin) anregt. TSH steigert die Produktion von T3 und T4. Hohe T3- und T4-Level führen zu einer negativen Rückkopplung und damit zur Verringerung der Produktion.

In der Schilddrüse wird wesentlich mehr T4 (ca. $80 \%$ ) als T3 (ca. 20\%) produziert. T3 ist die biologisch hauptsächlich wirk- same Form. T4 hat zwar auch eigene Wirkungen, dient aber überwiegend als Transportform und wird in den Zielzellen zu T3 umgewandelt. Die Umwandlung von T4 in T3 findet vor allem in Leber und Nieren, aber auch in Gehirn, Herz und Skelettmuskulatur statt.

Die Schilddrüse und ihre Hormone interagieren mit allen Organsystemen und Geweben des Körpers [3]. Störungen der Schilddrüsenfunktion können daher zu einer Vielzahl unterschiedlicher Symptome führen. Keines davon tritt ausschließlich bei einer Hypothyreose auf.

\section{Formen der Hypothyreose}

Man unterscheidet zwischen 4 verschiedenen Formen der Hypothyreose ( $\triangleright$ Tab. 1). Liegt die Ursache in der Schilddrüse selbst, handelt es sich um eine primäre Hypothyreose. Die Ursache kann eine idiopathische Follikelatrophie oder eine Entzündung infolge einer Verletzung oder einer Autoimmunerkrankung sein. $\mathrm{Zu}$ wenig Selen oder Zink und $\mathrm{zu}$ viel oder zu wenig Jod in der Nahrung sowie eine altersbedingte Atrophie können ebenfalls zu einer primären Hypothyreose führen.

Liegt eine Störung der übergeordneten Steuerung in der Hypophyse vor, handelt es sich um eine sekundäre Hypothyreose. Bei einer Störung im Bereich des Hypothalamus spricht man von einer tertiären Hypothyreose.

Darüber hinaus kann es zu einer Unterdrückung der Schilddrüse durch andere Erkrankungen kommen. Man spricht dann von euthyreot kranken Tieren. Zum Teil wird auch der Begriff NTI, englisch für „non thyroidal illness“ gebraucht. Stress und bestimmte Medikamente können ebenfalls zu einer Unterdrückung der Schilddrüse führen. Hierbei sind vor allem Kortison, Phenobarbital und Sulfonamide zu nennen.

\section{Autoimmune Thyreoiditis \\ Häufigkeit}

Die autoimmune Thyreoiditis ist die häufigste Stoffwechselerkrankung beim Hund. Die Angaben zur Häufigkeit des Auftretens schwanken je nach Autor zwischen 1:120-1:500. Die Angaben beziehen sich jeweils auf die Häufigkeit des Nachweises der Erkrankung im jeweiligen Spezialgebiet des Autors, z.B. Hautprobleme, Herz-Kreislauf-Erkrankungen oder neurologische Erkrankungen. Daher

Tab. 1 Hypothyreose und Verhaltensauffälligkeiten.

\begin{tabular}{|l|l|}
\hline Schilddrüsenunterfunktion & Ursachen \\
\hline primäre Hypothyreose & $\begin{array}{l}\text { - ideopathische Follikelatrophie } \\
\text { - Thyreoiditis, autoimmun oder nach Verletzungen der } \\
\text { Schilddrüse }\end{array}$ \\
& - Jodungleichgewicht, Mangel an Zink oder Selen \\
& - Altersatrophie \\
\hline sekundäre Hypothyreose & Störungen der übergeordneten Steuerung in der Hypophyse \\
\hline tertiäre Hypothyreose & Störungen der übergeordneten Steuerung im Hypothalamus \\
\hline Unterdrückung der & - Stress \\
\hline Schilddrüsenfunktion & - Krankheiten (non thyroidal illness) \\
& - Medikamente, z. B. Kortison, Phenobarbital, Sulfonamide
\end{tabular}




\section{Häufig betroffene Rassen}

Die Aufzählung gibt einen Überblick über die am häufigsten betroffenen Rassen, die in der Praxis der Autorin mit überwiegend subklinischer, teilweise auch klinischer Hypothyreose vorgestellt werden:

- American Staffordshire Terrier

- Australian Shepherd

- Bearded Collie

- Beauceron

- Berger de Pyrénées

- Berner Sennenhund

- Border Collie

- Briard

- Deutsche Dogge

- Deutscher Schäferhund

- Dobermann

- Eurasier

- Flat Coated Retriever

- Golden Retriever

- Hovawart

- Irish Setter

- Jack Russel Terrier

- Labrador Retriever

- kleiner und großer Münsterländer

- Parson Russell Terrier

- Rhodesian Ridgeback

- Rottweiler

- großer Schweizer Sennenhund

- Ungarischer Vizsla

- Weimaraner

- West Highland Terrier

Die Liste erhebt keinen Anspruch auf Vollständigkeit.

ist die tatsächliche Häufigkeit in der Hundepopulation vermutlich deutlich höher anzusetzen. Einer Studie zufolge waren bei einem Sechstel der Hunde, die in spezialisierten deutschen Tierarztpraxen zur Verhaltenstherapie vorgestellt wurden, somatische Ursachen nachweisbar [4]. An erster Stelle standen klinische und subklinische Hypothyreosen.

\section{Ätiologie}

Die autoimmune Thyreoiditis des Hundes ist vergleichbar mit der HashimotoErkrankung des Menschen.

Für den Dobermann, Englischen Setter und Rhodesian Ridgeback wurde bereits - wie beim Menschen - eine Verbindung mit den Genen für den Haupthistokompatibilitätskomplex nachgewiesen. Ob die genetische Veranlagung alleine zum Ausbrechen der Erkrankung führt oder ob zusätzliche Faktoren dazu nötig sind, ist umstritten. Betroffene Hunde haben eine erhöhte Wahrscheinlichkeit weitere autoimmune Erkrankungen zu entwickeln.

\section{Symptome}

Allergien und paradoxe Verhaltenssymptome entwickeln sich häufig mit der Pubertät, deutliche körperliche Symptome hingegen erst im Alter von 3-5 Jahren. Eine Übersicht der häufigsten Symptome findet sich in $>$ Tab. 2 .

\section{konkret}

Das „klassische“ Bild einer Hypothyreose, wie es in den Lehrbüchern zu finden ist, entsteht erst, wenn mehr als $75 \%$ des Schilddrüsengewebes zerstört sind.

\section{Verhaltensauffälligkeiten}

Hypothyreosepatienten mit Verhaltensauffälligkeiten zeigen überwiegend pa-

\section{Exkurs}

\section{Hashimoto-Erkrankung}

Die 1912 von dem japanischen Arzt Hakaru Hashimoto zuerst beschriebene chronische lymphozytäre Thyreoiditis beim Menschen ist eine Autoimmunerkrankung, die familiär gehäuft auftritt. Dabei wird das Schilddrüsengewebe durch T-Lymphozyten zerstört und es kommt zur Bildung von Antikörpern gegen schilddrüsenspezifische Antigene. Auf Dauer führt dies zu einer Hypothyreose. Bedingt durch die Zerstörung des Schilddrüsengewebes kann es aber zwischenzeitlich zu hyperthyreoten Phasen kommen.

radoxe Symptome, also solche, die man eigentlich bei einer Hyperthyreose erwarten würde. Sie sind meist hektisch, nervös und hyperaktiv, einige zeigen aber auch depressives Verhalten. Sie sind oft launisch, haben gute und schlechte Tage oder zeigen innerhalb weniger Stunden starke Verhaltensschwankungen. Extreme Unruhe und exzessive Lautäußerungen können auftreten [1].

Tab. 2 Häufige Symptome bei subklinischer Hypothyreose.

\begin{tabular}{|c|c|}
\hline subklinische Hypothyreose & Symptome \\
\hline Verhaltenssymptome & $\begin{array}{l}\text { - hyperaktiv, hektisch, nervös } \\
\text { - launisch } \\
\text { - geringe Stresstoleranz } \\
\text { - geringe Frustrationstoleranz } \\
\text { ängstlich und/oder aggressiv (z. T. anfallsartig oder mit } \\
\text { völligem Kontrollverlust) } \\
\text { - Konzentrations- und Lernschwäche } \\
\text { - evtl. depressiv }\end{array}$ \\
\hline körperliche Symptome & $\begin{array}{l}\text { - } \text { wärmeliebend, hitzeintolerant } \\
\text { - unspezifische Hautsymptome, Allergien, Otitiden, } \\
\text { Demodikose } \\
\text { - stumpfes Fell, Schuppen, „Babypelz“ } \\
\text { - Schwarzfärbung der Haut, Haarausfall (oft bilateral } \\
\text { symmetrisch) } \\
\text { - Gewichtsprobleme (Übergewicht, evtl. sehr dünn) } \\
\text { - Myxödem } \\
\text { - unspeurologische Erkrankungen, Epilepsie } \\
\text { - Leistungsschwäche } \\
\text { - Herz-Kreislauf-Erkrankungen } \\
\text { - Magen-Darm-Probleme } \\
\text { Willebrand-Erkrankung } \\
\text { - gestörter Sexualzyklus, mangelhafte Libido, Fruchtbar- } \\
\text { keitsstörungen } \\
\text { - vorzeitige Vergreisung }\end{array}$ \\
\hline
\end{tabular}


Die Patienten haben eine geringe Stressund Frustrationstoleranz, können sich häufig schlecht konzentrieren und zeigen zum Teil ausgeprägte Lernschwächen. Gesteigerte Ängstlichkeit, insbesondere Geräuschängste und Angst vor Gewitter, aber auch verstärkte Reizbarkeit und Aggression, z.T. anfallsartig oder mit vollständigem Kontrollverlust, sind typisch bei Schilddrüsenpatienten. Bei einigen Hypothyreosepatienten ist Geräuschangst das erste und eventuell für lange Zeit auch das einzige erkennbare Symptom. Untersuchungen an der Ludwig-Maximilians-Universität zeigten einen eindeutigen Zusammenhang zwischen subklinischer Hypothyreose und Geräuschangst bei untersuchten Bearded Collies [5].

\section{Körperliche Symptome}

Nicht alle Hypothyreosepatienten sind verhaltensauffällig, viele entwickeln nur körperliche Symptome. Hunde, die zunächst nur Verhaltenssymptome zeigen, entwickeln bei fortschreitender Erkrankung zunehmend auch körperliche Symptome, wenn sie unbehandelt bleiben. Sie kommen morgens schwer in Gang, sind wärmeliebend, aber gleichzeitig hitzeintolerant. Sie frieren schnell, liegen gerne direkt an der Heizung oder im Sommer in der prallen Sonne, sind aber bei höheren Temperaturen körperlich kaum belastbar.

Typisch sind unspezifische Hautsymptome, Allergien, Otitiden, Demodikose, stumpfes Fell, Schuppenbildung und „Babypelz“. Langhaarige Hunde sehen oft aus wie ein „explodierter Handfeger“. Haarausfall, der oft bilateral symmetrisch auftritt und besonders an den Schenkelinnenseiten und am Bauch lokalisiert ist sowie Schwarzfärbungen der Haut sind ebenfalls typisch.

Viele Schilddrüsenpatienten haben Gewichtsprobleme. Meistens neigen sie selbst bei restriktiver Fütterung - zu Übergewicht. Im Einzelfall treten auch hier paradoxe Symptome auf und die Hunde sind trotz intensiver und hochwertiger Fütterung extrem dünn. Substituiert man sie, nehmen sie zu und der Futterbedarf normalisiert sich.
Ein typisches, wenn auch selteneres Symptom, ist die Ausbildung eines Myxödems. Dies kann nur am Kopf oder am ganzen Körper auftreten und führt zu einem aufgequollenen, feisten Aussehen. Der Körperstamm wirkt tonnenförmig wie bei einem Aberdeen-Angus-Rind.

Häufiger treten neurologische Symptome auf, beispielsweise:

- Schluckbeschwerden

- Fazialislähmung

- Megaösophagus

- steifer Gang

- Harninkontinenz

- Muskelzittern

- Absencen

- fokale Anfälle

- Epilepsie (Grand-Mal-Anfall)

Es können unspezifische Schmerzen und Lahmheiten auftreten.

Viele Patienten zeigen eine ausgeprägte Leistungsschwäche, die durch eine reduzierte Sauerstoffbindungskapazität der Erythrozyten bedingt ist, sowie eine Bradykardie. In der Folge können sich Herz-Kreislauf-Erkrankungen entwickeln. Außerdem kann eine verstärkte Blutungsneigung infolge von Blutbildveränderungen, oft auch in Kombination mit der von-Willebrand-Erkrankung auftreten [2].

Im Zusammenhang mit einer Schilddrüsenunterfunktion sind häufig auch Magen-Darm-Probleme zu beobachten. Störungen der Sexualfunktionen können bei beiden Geschlechtern auftreten. Unfruchtbarkeit und mangelnde Libido beim Rüden sowie verlängerter Interöstrus, ausbleibende Läufigkeit und stille Hitze bei der Hündin sind typische Phänomene. Ein Teil der betroffenen Tiere zeigt eine ausgeprägte vorzeitige Vergreisung. Die Hunde können bereits im Alter von 2 oder 3 Jahren stark ergrauen und wirken vom gesamten Habitus wie ein uralter Hund.

\section{Diagnostik}

Bei Hunden mit Verhaltensauffälligkeiten muss eine ausführliche Verhaltensanamnese erhoben werden. Da jede Erkrankung zu einer Unterdrückung der 


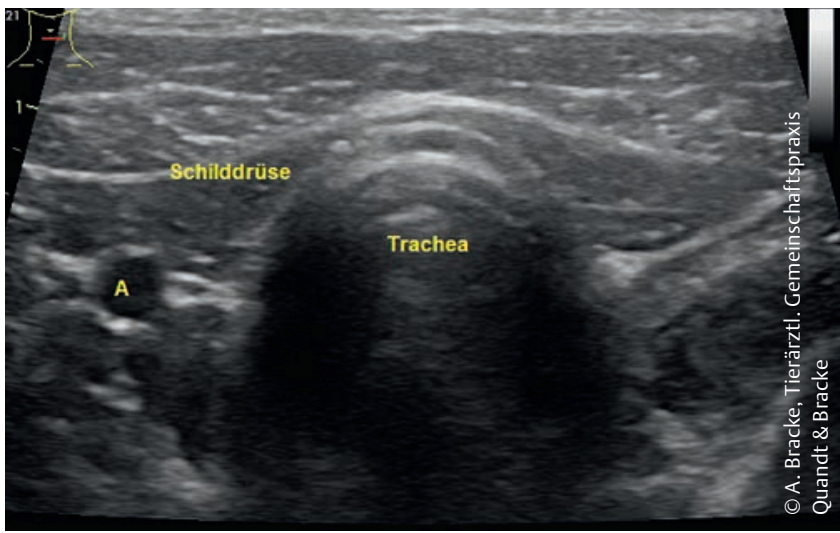

Abb. 1 Ultraschall der Schilddrüse: Normales Schilddrüsengewebe bei einer 18 Monate alten Labradorhündin ( $\mathrm{A}=\mathrm{A}$. carotis externa).

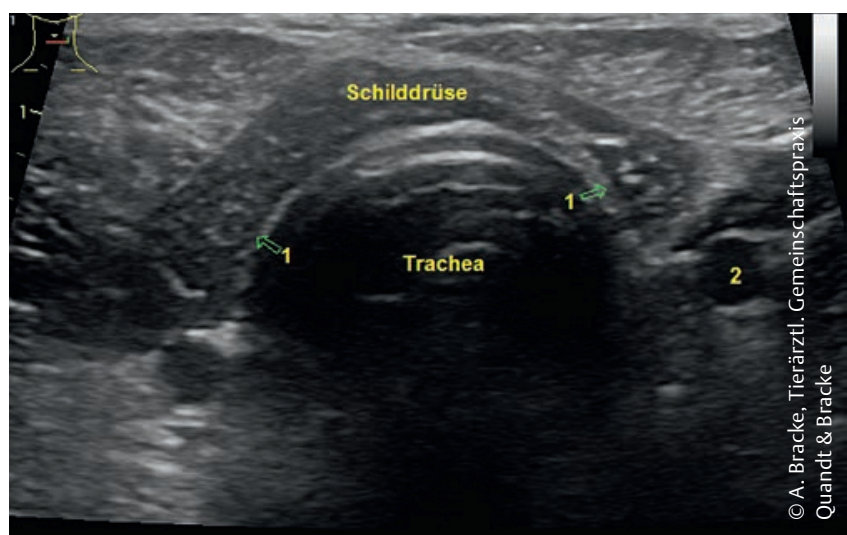

Abb. 2 Ultraschall der Schilddrüse: Verändertes Schilddrüsengewebe bei einer 10-jährigen Labradorhündin mit frühzeitig behandelter autoimmuner Thyreoiditis. Ausbruch der Erkrankung mit 4 Jahren $(1=$ verändertes Schilddrüsengewebe, 2 = A. carotis externa). Nähere Informationen zu dem Fall bei der Autorin.
Schilddrüsenfunktion führen kann, sollten in jedem Fall ein Organprofil und ein großes Blutbild gemacht werden.

Bei Hunden, die mit selbst zusammengestellten Rationen gefüttert werden, sollten zudem Jod, Selen und Zink überprüft werden.

Darüber hinaus sollten die folgenden Schilddrüsenwerte untersucht werden:

- fT4 (freies T4)

- $\mathrm{T} 4$

- fT3 (freies T3)

- T3

- $\mathrm{TSH}$

- TAK (Thyreoglobulin-Antikörper), z.T. auch als TGA bzw. TGAA (Thyreoglobulin-Autoantikörper) bezeichnet

- T4-Autoantikörper

- T3-Autoantikörper

- k-Wert (Korrelationswert fT4-Cholesterin)

Außerdem kann ein TRH-Stimulationstest sinnvoll sein.

Bei fortgeschrittenen Schilddrüsenerkrankungen sind im Ultraschall die Veränderungen im Schilddrüsengewebe erkennbar ( $\triangleright$ Abb. 1, $\triangleright$ Abb.2) [6]. Die Ermittlung der Herzfrequenz in Ruhe und nach Belastung liefert oft weitere wertvolle Hinweise. Im Einzelfall sollten - je nach Symptomatik - folgende Untersuchungen durchgeführt werden:

- Herzultraschall

- EKG
- Blutdruckmessung

- neurologische Untersuchung

- orthopädische Untersuchung

- Schmerzdiagnostik

- Untersuchungen auf Borreliose, Ehrlichiose usw.

\section{Autoimmune Thyreoiditis}

Die autoimmune Thyreoiditis verläuft in der Regel schleichend. Oft zeigen die Laborwerte erst dann eine eindeutige Diagnose, wenn bereits ein Großteil der Schilddrüse zerstört ist. Bis dahin verläuft die Krankheit eventuell jahrelang subklinisch. Da die Symptome häufig sehr unspezifisch sind, werden sie isoliert betrachtet und mit wechselndem Erfolg behandelt, ohne dass die eigentliche Ursache erkannt wird. Gerade bei Hunden, die nur Verhaltensauffälligkeiten zeigen, wird die Problematik gerne dem individuellen Charakter, der Rasse oder der Unfähigkeit des Besitzers zur Erziehung seines Hundes angelastet. Erschwert wird die diagnostische Zuordnung auch dadurch, dass die typischen Verhaltensprobleme, die bei Schilddrüsenpatienten auftreten, durchaus auch andere Ursachen haben können.

Um die Diagnose subklinische Hypothyreose zu einem möglichst frühen Zeitpunkt stellen zu können, müssen möglichst viele diagnostische Kriterien herangezogen werden.

Zudem können so durch rechtzeitige Behandlung körperliche Folgeschäden und durch Lernprozesse langfristig zementierte unerwünschte Verhaltensweisen vermieden werden.

Bei Folgeuntersuchungen sollte möglichst immer dasselbe Labor benutzt werden, da die Untersuchungsmethoden und Normwerte der verschiedenen Labors nicht vergleichbar sind. Ein großes Schilddrüsenprofil, mit allen oben genannten Werten, wird zurzeit in Deutschland nur von Laboklin und Idexx angeboten.

Typische Laborveränderungen bei subklinischer Hypothyreose sind:

- erhöhter Cholesterinspiegel

- hoher MCHC- und MCH-Spiegel

- Eosinophilie

- Thrombozytopenie

- erhöhte Leber- oder Nierenwerte

\section{Auswertung des Schilddrüsen- profils}

\section{konkret}

Nach der Verabreichung von Medikamenten, die die Schilddrüse beeinflussen, können aussagefähige Werte des Schilddrüsenprofils frühestens 4 Wochen nach Ende der Behandlung ermittelt werden.

Die folgenden Aussagen gelten für Hunde, bei denen keine andere Erkrankung vorliegt, die zu einer Unterdrückung der Schilddrüsenfunktion führt. 


\section{Wissenswert}

Auch beim Hund gibt es Schilddrüsenpatienten mit Umwandlungsstörungen, die selbst bei ausreichendem T4-Level nicht in der Lage sind, genug T3 herzustellen. Diese Patienten müssen zusätzlich mit T3 substituiert werden. Hunde mit Umwandlungsstörungen sind oft massiv verhaltensauffällig oder zeigen besonders schwere neurologische Symptome.

Da es keine für den Hund zugelassenen T3-Präparate gibt, muss für diese Patienten das humanmedizinische Thybon ${ }^{\circledR}$ umgewidmet werden. Die Einstiegsdosis liegt bei $1,1 \mu \mathrm{g} / \mathrm{kg} 2$-mal täglich. Nach 1 Woche wird auf die empfohlene Dosis von 2,2 $\mathrm{\mu g} / \mathrm{kg}$ 2-mal täglich erhöht. Im Einzelfall muss mittags eine 3. Dosis verabreicht werden, wenn sich das Befinden im Laufe des Tages deutlich verschlechtert. Bitte beachten Sie, dass bei T3-Substitution die empfohlene Dosis wegen der kurzen Halbwertszeit mehrmals täglich verabreicht werden muss; also jeweils $2,2 \mu \mathrm{g} / \mathrm{kg}$.

\section{TSH}

Ist der TSH-Wert erhöht, kann man von einer Hypothyreose ausgehen. Da beim Hund das TSH sehr schnell gegenreguliert wird, ist ein normaler TSH-Wert aber leider keine Garantie für eine gesunde Schilddrüse oder eine passende Einstellung bei der Substitution.

\section{Thyreoglobulin-Antikörper bzw. T3-/T4-Antikörper}

Sind Thyreoglobulin-Antikörper bzw. T3-/T4-Antikörper vorhanden, handelt es sich um eine autoimmune Thyreoiditis. Allerdings sind nur bei einem Teil der betroffenen Patienten bzw. nur zeitweise Antikörper nachweisbar. Fehlende Antikörper sind daher kein Beweis für eine gesunde Schilddrüse. Beim Vorhandensein von T3-/T4-Antikörpern kann es zu paradox hohen Hormonwerten kommen, was eine diagnostische Zuordnung erschwert.

Vom Labor wird für T4-Autoantikörper ein Normbereich bis $20 \%$ angegeben, für T3-Autoantikörper bis 10\%. Alle Patienten, die die Autorin in den letzten Jahren betreut hat, bei denen Antikörper gegen die Hormone - auch im niedrig „normalen“ Bereich - nachweisbar waren, entwickelten spätestens innerhalb 1 Jahres eine manifeste Hypothyreose. Es erscheint auch wenig logisch, dass überhaupt Antikörper gegen ein körpereigenes Hormon vorhanden sein sollten.

\section{fT3, T3, fT4, T4}

Liegen die Hormonwerte unterhalb der Labornorm, liegt eine Hypothyreose vor.
Wenn bei jungen Hunden mit passender Symptomatik die Hormone im unteren Drittel des Normbereichs sind, liegt höchstwahrscheinlich eine subklinische Hypothyreose vor. Findet man bei jungen Hunden mit passender Symptomatik Hormonwerte in der unteren Hälfte des Normbereichs, handelt es sich wahrscheinlich um eine subklinische Hypothyreose.

\section{k-Wert}

Ist der k-Wert $<-4$, liegt eine Hypothyreose vor. Bei einem k-Wert zwischen -4 und +1 (fraglicher Wert) ist gegebenenfalls ein TRH-Stimulationstest durchzuführen.

\section{TRH-Stimulationstest}

Besteht der Verdacht einer subklinischen Hypothyreose, kann dieser eventuell durch einen TRH-Stimulationstest erhärtet werden. Aber auch hier ergibt sich nicht immer eine eindeutige Aussage. Sind keine Antikörper vorhanden und gibt es andere mögliche Erklärungen für die Symptomatik bzw. die niedrigen Schilddrüsenwerte, sollten diese zunächst behandelt werden. Dies ist insbesondere dann der Fall, wenn andere, eventuell auch vorausgegangene Erkrankungen bekannt sind, der Hund ein Deprivationssyndrom hat oder unter starkem bzw. schon lange anhaltendem Stress steht. Verschwinden die Symptome nicht, empfiehlt es sich, die Schilddrüse nach 3-6 Monaten noch einmal zu untersuchen. Bei Patienten mit Verdacht auf eine subklinische Hypothyreose, bei denen keine anderen möglichen Ursa- 
chen für die Symptome erkennbar sind, sollte eine diagnostische Substitution durchgeführt werden.

\section{Therapie}

\section{Dosierung}

Die meisten Hunde mit einer Hypothyreose lassen sich mit einer Tagesdosis von $20-30 \mu \mathrm{g} / \mathrm{kg}$ Thyroxin, z.B. Forthyron ${ }^{\circledR}$ oder Wethyrox ${ }^{\circledR}$, verteilt auf 2-mal täglich, gut einstellen. Im Einzelfall kann aber auch weniger oder deutlich mehr gebraucht werden. Für Patienten, deren Besitzer Probleme haben 2-mal am Tag Medikamente zu geben, kann Leventa ${ }^{\circledR}$ eine Alternative sein.

Bei Hunden mit subklinischer Hypothyreose und bei altersbedingter Atrophie hat sich eine Einstiegsdosis von 2-mal täglich $5 \mu \mathrm{g} / \mathrm{kg}$ bewährt. Je nach Wirkung sollte die Dosis dann wöchentlich um 1-2 $\mu \mathrm{g} / \mathrm{kg}$ gesteigert werden, bis die Wohlfühldosis erreicht ist. Nach 4-6 Wochen unter dieser Dosierung sollte eine Substitutionskontrolle erfolgen, um den persönlichen Referenzwert für die Zukunft zu ermitteln. Kommt es bis zum Erreichen der empfohlenen Höchstdosis von $30 \mu \mathrm{g} / \mathrm{kg}$ am Tag nicht zu einer befriedigenden Verbesserung der Symptome, sollte eine Kontrolluntersuchung erfolgen.

Ist der T4-Wert am oberen Ende der Norm oder darüber, ist auf jeden Fall der T3-Wert zu überprüfen. Ansonsten sollte die Dosis von Forthyron ${ }^{\circledR}$ entsprechend weiter erhöht werden. Die Blutentnahme für die Kontrolluntersuchungen sollte 4-6 Stunden nach Tablettengabe erfolgen.

\section{Applikation}

Bei Verabreichung mit dem Futter reduziert sich die Resorption der Hormone erheblich.

Thyroxin und Trijodthyronin sollten daher auf nüchternen Magen gegeben und in etwas Wurst, Käse oder Feuchtfutter verpackt werden. Die Verabreichung sollte mindestens $30 \mathrm{Mi}$ nuten vor der Fütterung erfolgen.

Bei ausreichender Substitution stellt die Schilddrüse ihre Produktion vollständig ein. Beim Hund wird dadurch der Autoimmunprozess gebremst, im günstigsten Fall sogar vollständig gestoppt. Es gibt aber unterschiedliche Verlaufsformen. Einige Patienten lassen sich innerhalb weniger Wochen gut einstellen und sind damit jahrelang stabil, andere haben immer wieder akute Schübe und müssen dann wieder neu eingestellt werden. Insbesondere Patienten mit Antikörpern gegen T3 bzw. T4 brauchen eventuell zeitweise das 2- bis 3fache der empfohlenen Dosis, um vernünftige Blutwerte zu erreichen.

Gelingt es, die Antikörperproduktion zum Stillstand zu bringen, reduziert sich der Tablettenbedarf auf ein normales Niveau. Die Behandlung mit Schilddrüsenhormonen - auch über längere Zeit - führt nicht zu einer Schädigung der Schilddrüse. Liegt keine primäre Schilddrüsenerkrankung vor, nimmt die Schilddrüse ihre Arbeit wieder auf, sobald die Substitution beendet wird. Das Absetzen der Schilddrüsentabletten nach einer länge- 
ren Substitution, beispielsweise zur symptomatischen Behandlung bei euthyreot kranken Tieren, sollte nicht abrupt erfolgen. Um einen sanften Übergang zu ermöglichen, sollten die Tabletten ausgeschlichen werden. Liegt eine primäre Schilddrüsenerkrankung vor, müssen die Patienten lebenslang substituiert werden.

\section{Fazit}

Subklinische und manchmal auch klinische Hypothyreosen beim Hund werden häufig nicht - oder erst sehr spät - diagnostiziert. Einerseits, weil die Symptome vielfältig, individuell sehr unterschiedlich ausgeprägt und größtenteils unspezifisch sind. Andererseits, weil die angegebenen Normwerte im unteren Bereich für viele Hunde nicht stimmen. Es gibt Hunde, die mit einem T4-Wert von $2,4 \mu \mathrm{g} / \mathrm{kg}$ gesund sind und andere, die damit bereits massive klinische Symptome haben. Insgesamt wird die subklinische Hypothyreose beim Hund nach den Erfahrungen der Autorin eher unterdiagnostiziert. Allerdings gibt es auch Fälle, bei denen auf Druck der Tierbesitzer gesunde Hunde substituiert werden, weil sie ein Verhalten zeigen, das den Besitzern nicht gefällt. Eine diagnostische Therapie fügt der Schilddrüse keinen Schaden zu, sollte aber kritisch evaluiert werden, wenn keine Besserung eintritt.

Allen, die sich intensiver mit den physiologischen Zusammenhängen zwischen Schilddrüsenfunktion und Verhalten beschäftigen möchten, sei das Buch von Zimmermann [7] als Lektüre empfohlen.

\section{Online zu finden unter}

http://dx.doi.org/10.1055/s-0042-113537

\section{Literatur}

1 Dodds WJ. Behavioral changes associated with thyroid dysfunction in dogs. Proceedings Am Hol Vet Med Assoc 1999; 80-82

2 Dodds WJ. Bleeding disorders in animals: hereditary disorders. Proceedings of $30^{\text {th }}$ WSAVA World Congress Mexico; 2005
3 Heldmaier G, Neuweiler G, Rössler W. Vergleichende Tierphysiologie. Band 2. Vegetative Physiologie. Berlin: Springer; 2003

4 Köhler K. Evaluierung von somatischen Ursachen für Verhaltensveränderungen beim Hund in der tierärztlichen Praxis [Dissertation]. München: Tierärztliche Fakultät der Ludwig-Maximilians-Universität München; 2005

5 Lauinger I. Geräuschempfindlichkeit beim Hund am Beispiel des Bearded Collies - Ein Vergleich von Verhaltenstherapiemaßnahmen und Substitution mit Thyroxin [Dissertation]. München: Tierärztliche Fakultät der Ludwig-Maximilians-Universität München; 2012

6 Wisner E, Nylon TG. Ultrasonography of the thyroid and parathyroid glands. Vet Clin North Am Small Anim 1998; 28 (4): 973-991

7 Zimmermann B. Schilddrüse und Verhalten. 4. Aufl. Zossen: MenschHund; 2012

\section{Christiane Wergowski}

Freiholzer Weg 1

18516 Süderholz

www.christiane-wergowski.de christiane.wergowski@web.de 